\title{
ASYMPTOTIC BEHAVIOR OF SOLUTIONS OF SECOND ORDER NONLINEAR DIFFERENCE EQUATIONS*
}

\author{
Jizhong WANG, FANWei Meng ANd Jibao Li
}

\begin{abstract}
In this paper, we study the asymptotic behavior of the second order difference equation

$(*)$

$$
\Delta(r(n) \Delta x(n))+f(n, x(n))=0 .
$$

we obtain some sufficient conditions which ensure that all the solutions of $(*)$ are bounded, and also obtain some conditions which guarantee that for every solution $x(n)$ of $(*)$ satisfies $|x(n)|=O\left(R\left(n, n_{0}\right)\right)$ as $n \rightarrow \infty$, where $R(n, s)=\sum_{k=s}^{n-1} \frac{1}{r(k)}$.
\end{abstract}

\section{A discrete inequality}

In the sequel we will require the following discrete inequality which extends the known discrete inequality obtained by Meng [5].

DEFInItion. A function $g(u)$ is said to belong to $\mathscr{I}$ if $g(u)$ is nondecreasing and continuous on $(0, \infty)$ and

$$
g(u) / v \leqq g(u / v), \quad u \geqq 0, v \geqq 1 .
$$

Every where we mean that $\sum_{k=s}^{n} \alpha(k)=0$ if $n<s$.

LEMMA. Let $x(n), h_{i}(n), i=1,2, \cdots, m$ be real valued nonnegative functions defined on $N\left(n_{0}\right)=\left\{n_{0}, n_{0}+1, \cdots\right\}, n_{0} \in\{1,2, \cdots\}, f(n) \geqq 1$ be nondecreasing on $N\left(n_{0}\right), g_{i}(u) \in \mathscr{F}, i=1,2, \cdots, m$. Suppose that the discrete inequality

1980 Mathematics Subject Classification (1985 Revision). Primary : 39A10.

Key words and phrases. discrete inequality, difference equation, asymptotic behavior of solutions.

* The Subject is Supported by the Natural Science Foundation of Shandong Province P.R. China.

Received March 2, 1995. 


$$
x(n) \leqq f(n)+\sum_{i=1}^{m}\left(\sum_{s=n_{0}}^{n-1} h_{i}(s) g_{i}(x(s))\right)
$$

holds for all $n \in N\left(n_{0}\right)$. Then we have

$$
x(n) \leqq f(n) \prod_{i=1}^{m} E_{i}(n), \quad n \in N\left(n_{0}\right) .
$$

Where $G_{k}(u)=\int_{u_{0}}^{u} \frac{1}{g_{k}(s)} d s, 0<u_{0}, u \geqq 0$,

$$
E_{k}(n)=G_{k}^{-1}\left[G_{k}(1)+\sum_{s=n_{0}}^{n-1} h_{k}(s) \prod_{i=1}^{k-1} E_{i}(s)\right], \quad k=1,2, \cdots, m .
$$

$G_{k}^{-1}$ is the inverse of $G_{k}$. Here it is supposed that

$$
\prod_{i=1}^{0} E_{i}(n)=1, \quad n \in N\left(n_{0}\right) .
$$

Proof. The proof is by Mathematical induction. We first suppose that here $m=1$. Since $f(n)$ is nondecreasing, $g_{1} \in \mathscr{F}$ and $f(n) \geqq 1$, we have from (1)

$$
\begin{aligned}
\frac{x(n)}{f(n)} & \leqq 1+\sum_{s=n_{0}}^{n-1} \frac{h_{1}(s)}{f(s)} g_{1}(x(s)) \\
& \leqq 1+\sum_{s=n_{0}}^{n-1} h_{1}(s) g_{1}\left(\frac{x(s)}{f(s)}\right) .
\end{aligned}
$$

Now using the discrete Bihari's inequality [4], we obtain

$$
x(n) \leqq f(n) E_{1}(n), \quad n \in N\left(n_{0}\right),
$$

where

$$
E_{1}(n)=G_{1}^{-1}\left[G_{1}(1)+\sum_{s=n_{0}}^{n-1} h_{1}(s)\right]
$$

This proves the (2) is true for $m=1$. Now suppose that (2) is true for $m=k$. Then for $m=k+1$ we may rewrite the inequality (1) as

$$
x(n) \leqq f_{1}(n)+\sum_{i=1}^{k}\left(\sum_{s=n_{0}}^{n-1} h_{i}(s) g_{i}(x(s))\right), \quad n \in N\left(n_{0}\right),
$$

where

$$
f_{1}(n)=f(n)+\sum_{s=n_{0}}^{n-1} h_{k+1}(s) g_{k+1}(x(s)) .
$$

Obviously, here $f_{1}(n)$ satisfies the condition for $f(n)$, so by the inductive assumption we obtain from (5)

$$
x(n) \leqq f(n) R(n)+R(n)\left(\sum_{s=n_{0}}^{n-1} h_{k+1}(s) g_{k+1}(x(s))\right)
$$


where

$$
R(n)=\prod_{i=1}^{k} E_{i}(n)
$$

So we have

$$
\frac{x(n)}{f(n) R(n)} \leqq 1+\sum_{s=n_{0}}^{n-1} h_{k+1}(s) R(s) g_{k+1}\left(\frac{x(s)}{f(s) R(s)}\right) .
$$

This inequality is of the form (3), and hence by the first step of our proof we get from (6)

$$
\begin{aligned}
x(n) & \leqq f(n) R(n) G_{k+1}^{-1}\left[G_{k+1}(1)+\sum_{s=n_{0}}^{n-1} h_{k+1}(s) R(s)\right] \\
& =f(n) \prod_{i=1}^{k+1} E_{i}(n), \quad n \in N\left(n_{0}\right) .
\end{aligned}
$$

This proves that (2) is true for $m=k+1$. The proof is complete.

Example. The following example illustrates the Lemma. Let

$$
x(n) \leqq f(n)+\sum_{\imath=1}^{m}\left(\sum_{s=n_{0}}^{n-1} h_{i}(s)[x(s)]^{r_{\imath}}\right)
$$

where $0<r_{i}<1, i=1,2, \cdots, m$. We note that $x^{r_{\imath} \in \mathcal{F}}$ and $x, f, h_{\imath}(i=1,2, \cdots, m)$ are defined as in Lemma. It is easy to observe

and

$$
G_{i}(u)=\frac{1}{1-r_{\imath}}\left[u^{1-r_{\imath}}-u_{0}^{1-r_{\imath}}\right], \quad \imath=1,2, \cdots, m, 0<r_{i}<1,
$$

$$
G_{\imath}^{-1}(u)=\left[\left(1-r_{\imath}\right) u+u_{0}^{1-r_{\imath}}\right]^{1 /\left(1-r_{i}\right)}, \quad \imath=1,2, \cdots, m, \quad 0<r_{i}<1 .
$$

Similarly, we obtain

$$
\begin{aligned}
& E_{1}(n)=\left[1+\left(1-r_{1}\right) \sum_{s=n_{0}}^{n-1} h_{1}(s)\right]^{1 /\left(1-r_{1}\right)}, \\
& E_{i}(n)=\left[1+\left(1-r_{\imath}\right) \sum_{s=n_{0}}^{n-1} h_{i}(s) \prod_{k=1}^{2-1} E_{k}(s)\right]^{1 /\left(1-r_{i}\right)}, \quad i=2,3, \cdots, m .
\end{aligned}
$$

We conclude that

$$
x(n) \leqq f(n) \prod_{i=1}^{m} E_{i}(n), \quad n \in N\left(n_{0}\right)
$$

\section{Boundedness conditions}

We consider the following second order nonlinear difference equation

$$
\Delta(r(n) \Delta x(n))+f(n, x(n))=0, \quad n \in N\left(n_{0}\right),
$$

where $N\left(n_{0}\right)$ as in above Lemma, $\Delta$ is the forward difference operator, i.e. 
$\Delta x(n)=x(n+1)-x(n) . \quad r(n)$ is the real sequences and $f: N\left(n_{0}\right) \times R \rightarrow R(R$ is real line). With regard to the equation (7) we always assume that:

(H) $\quad r(n)>0$ for all $n \in N\left(n_{0}\right)$; in addition we always have

$$
|f(n, x)| \leqq \sum_{i=1}^{m} b_{i}(n) g_{i}(|x|)+b_{m+1}(n), \quad(n, x) \in N\left(n_{0}\right) \times R,
$$

where $b_{i}(n), \imath=1,2, \cdots, m+1$ are known nonnegative real functions on $N\left(n_{0}\right)$, $g_{i}(u) \in \mathscr{F}, i=1,2, \cdots, m$.

To simplity the notation in the sequel we will define

$$
R(n, s)=\sum_{k=s}^{n-1} \frac{1}{r(k)} \text {. }
$$

for any $s \in N\left(n_{0}\right)$ and all $n \in N(s+1)$. According to the limit of $R\left(n, n_{0}\right)$ as $n \rightarrow \infty$, there are two different case, namely:
(a) $\lim _{n \rightarrow \infty} R\left(n, n_{0}\right)<\infty$,
(b) $\lim _{n \rightarrow \infty} R\left(n, n_{0}\right)=\infty$.

Recently some results concerning the oscillatory and nonoscillatory properties of solutions of nonlinear difference equations of second order have been established in [1] (see also references therein).

The purpose of this paper is to present theorems that give sufficient conditions for all solutions of (7) to be bounded. Also, we obtain a result on the growth of solutions of (7). The obtained results are discrete analogues of some known theorems for nonlinear differential equations due to Yang [2] and Hatvanti [3].

We first establish some results for the case (a) as follows.

THEOREM 1. In addition to the hypothesses $(H)$, suppose further that

(i) $\sum_{s=n_{0}}^{n-1} b_{j}(s) R(n, s+1)$ is bounded on $N\left(n_{0}\right)$ for $1 \leqq \jmath \leqq m+1$;

(ii) $\lim _{n \rightarrow \infty} R\left(n, n_{0}\right)<\infty$.

Then every solution $x(n)$ of $(7)$ is bounded on $N\left(n_{0}\right)$.

Proof. Let $x(n)$ be a solution of (7). From (7) by successive summations, we obtain

$$
x(n)=x\left(n_{0}\right)+r\left(n_{0}\right) \Delta x\left(n_{0}\right) \sum_{k=n_{0}}^{n-1} \frac{1}{r(k)}-\sum_{k=n_{0}}^{n-1} \frac{1}{r(k)} \sum_{i=n_{0}}^{k-1} f(i, x(i)) .
$$

To exchange the order of the summation of the last term, we have

So

$$
x(n)=x\left(n_{0}\right)+r\left(n_{0}\right) \Delta x\left(n_{0}\right) \sum_{k=n_{0}}^{n-1} \frac{1}{r(k)}-\sum_{k=n_{0}}^{n-1} f(k, x(k)) R(n, k+1) .
$$

$$
|x(n)| \leqq a(n)+\sum_{i=1}^{m} \sum_{k=n_{0}}^{n-1} R(n, k+1) b_{i}(k) g_{i}\left(\left|x_{i}(k)\right|\right),
$$


where

$$
a(n)=1+\left|x\left(n_{0}\right)\right|+r\left(n_{0}\right)\left|\Delta x\left(n_{0}\right)\right| R\left(n, n_{0}\right)+\sum_{k=n_{0}}^{n-1} b_{m+1}(k) R(n, k+1) .
$$

By our Lemma, we obtain from above inequality

$$
|x(n)| \leqq a(n) \prod_{i=1}^{m} E_{i}(n), \quad n \in N\left(n_{0}\right) .
$$

Where

$$
E_{i}(n)=G_{i}^{-1}\left(G_{i}(1)+\sum_{s=n_{0}}^{n-1} b_{i}(s) R(n, s+1) \prod_{j=1}^{\imath-1} E_{j}(s)\right), \quad i=1,2, \cdots, m .
$$

$G_{\imath}, G_{\imath}^{-1}$ are defined as in Lemma. By the conditions (i) and (ii) we have $a(n)$ and $E_{i}(n)$ are bounded on $N\left(n_{0}\right)$. From (8) we have $x(n)$ is bounded on $N\left(n_{0}\right)$. The proof is complete.

COROLLARY 1. In addition to $(H)$, suppose that the following conditions are satisfied:

(i) there exists a constant $K>0$ such that $r(n) \geqq K$ for $n \in N\left(n_{0}\right)$;

(ii) $\lim _{n \rightarrow \infty} R\left(n, n_{0}\right)=A<\infty$;

(iii) $\sum_{k=n_{0}}^{\infty} b_{i}(k)<\infty, i=1,2, \cdots, m+1$.

Then every solution $x(n)$ of $(7)$ is bounded on $N\left(n_{0}\right)$.

Proof. It is easily seen that the conditions (ii) and (iii) in Corollary 1 imply the condition (i) in Theorem 1.

Example 1. Consider the equation

$$
\Delta\left(e^{c n} \Delta x\right)+\sum_{i=1}^{m} q_{i}(n) x^{p_{i}}=0, \quad n \in N\left(n_{0}\right) .
$$

Here $c>0$ and $p_{i} \in(0,1]$ are constants, and $q_{i}(n)$ are real polynomials. Clearly, all of the conditions in Theorem 1 are satisfied for (9). Thus all solutions $x(n)$ of (9) are bounded on $N\left(n_{0}\right)$.

The following result is concerned with the case (b).

THEOREM 2. Suppose the hypotheses $(H)$ hold, suppose moreover that

(i) $\sum_{k=n_{0}}^{\infty} b_{m+1}(k)<\infty, \sum_{k=n_{0}}^{\infty} b_{i}(k) R\left(k, n_{0}\right)<\infty,(i=1,2, \cdots, m)$;

(ii) $\lim _{n \rightarrow \infty} R\left(n, n_{0}\right)=\infty$.

Then for every solution $x(n)$ of (7) satisfies

$$
|x(n)|=O\left(R\left(n, n_{0}\right)\right), \quad|r(n) \Delta x(n)|=O(1), \text { as } n \rightarrow \infty .
$$

Proof. Let $x(n)$ be a solution of (7). From (7), by successive summations, we obtain 


$$
r(n) \Delta x(n)=r\left(n_{0}\right) \Delta x\left(n_{0}\right)-\sum_{k=n_{0}}^{n-1} f(k, x(k))
$$

and

$$
x(n)=x\left(n_{0}\right)+r\left(n_{0}\right) \Delta x\left(n_{0}\right) R\left(n, n_{0}\right)-\sum_{k=n_{0}}^{n-1} \frac{1}{r(k)} \sum_{i=n_{0}}^{k-1} f(i, x(i)), \quad n \in N\left(n_{0}\right) .
$$

And, so

$$
|x(n)| \leqq C_{1} R\left(n, n_{0}\right)+\sum_{j=1}^{m} \sum_{k=n_{0}}^{n-1} \frac{1}{r(k)} \sum_{i=n_{0}}^{k-1} b_{j}(i) g_{j}(|x(i)|)+\sum_{k=n_{0}}^{n-1} \frac{1}{r(k)} \sum_{i=n_{0}}^{k-1} b_{m+1}(i) .
$$

For some constant $C_{1}>0$ and all $n \geqq n_{1} \in N\left(n_{0}\right)$. By the first condition in (i), there exists constant $C_{2}>0$ such that

$$
\sum_{k=n_{0}}^{n-1} \frac{1}{r(k)} \sum_{i=n_{0}}^{k-1} b_{m+1}(i) \leqq C_{2} R\left(n, n_{0}\right) .
$$

Since $\lim _{n \rightarrow \infty} R\left(n, n_{0}\right)=\infty, R\left(n, n_{0}\right) \geqq 1$, for $n \geqq n_{1}, R\left(n, n_{0}\right) \geqq R(n, s), s \geqq n_{0}$ we obtain

$$
|x(n)| \leqq C R\left(n, n_{0}\right)+R\left(n, n_{0}\right) \sum_{j=1}^{m} \sum_{k=n_{0}}^{n-1} b_{j}(k) g_{j}(|x(k)|) .
$$

For some constant $C \geqq 1$, and so

$$
\frac{|x(n)|}{R\left(n, n_{0}\right)} \leqq C+\sum_{j=1}^{m}\left(\sum_{k=n_{0}}^{n-1} b_{j}(k) R\left(k, n_{0}\right) g_{j}\left(\frac{|x(k)|}{R\left(k, n_{0}\right)}\right)\right) .
$$

Applying the Lemma to (11), we have

$$
\frac{|x(n)|}{R\left(n, n_{0}\right)} \leqq C \prod_{i=1}^{m} V_{i}(n), \quad n \geqq n_{1} .
$$

Where

$$
V_{i}(n)=G_{\imath}^{-1}\left(G_{i}(1)+\sum_{s=n_{0}}^{n-1} b_{i}(s) R\left(s, n_{0}\right)\left(\prod_{j=1}^{i-1} V_{j}(s)\right)\right), \quad i=1,2, \cdots, m .
$$

Now, letting $n \rightarrow \infty$ in (12) and in view of the conditions (i) and (ii), then we obtain the desired relation $|x(n)|=O\left(R\left(n, n_{0}\right)\right)$. Finaly, by (12) we derive from $(10)$

$$
r(n)|\Delta x(n)| \leqq r\left(n_{0}\right)\left|\Delta x\left(n_{0}\right)\right|+\sum_{k=n_{0}}^{n-1} b_{m+1}(k)+\sum_{i=1}^{m} g_{i}(M)\left(\sum_{k=n_{0}}^{n-1} b_{i}(k) R\left(k, n_{0}\right)\right)<\infty,
$$

where the number $M>0$ is an upper bound for $C \prod_{i=1}^{m} V_{i}(n)$ on $N\left(n_{0}\right)$. This complete the proof.

COROLLARY 2. We consider the equation

$$
x(n+2)-2 x(n+1)+x(n)+f(n, x(n))=0, \quad n \in N\left(n_{0}\right) .
$$

If the hypotheses $(\mathrm{H})$ and the following conditions are satisfied: 


$$
\sum_{k=n_{0}}^{\infty} b_{m+1}(k)<\infty, \sum_{k=n_{0}}^{\infty} b_{i}(k) k<\infty, \quad i=1,2, \cdots, m .
$$

Then all solutions $x(n)$ of (13) satisfy the relations: $|x(n)|=O(n)$ and $\mid x(n+1)-$ $x(n) \mid=O(1)$ as $n \rightarrow \infty$.

Remark. Theorem 2 generalizes Theorem 8 in [1].

Example 2. Consider the following equation

$$
x(n+2)-2 x(n+1)+x(n)+\sum_{i=1}^{m} c_{i}(n) x^{p_{i}}(n)=0, \quad n \in N\left(n_{0}\right) .
$$

Where $p_{i}$ are numbers from $(0,1]$, and $c_{i}(n)(i=1,2, \cdots, m)$ are real sequences. If

$$
\sum_{k=n_{0}}^{\infty}\left|c_{i}(k)\right| k<\infty, \quad i=1,2, \cdots, m .
$$

Then by Corollary 2, all of the solutions of the equation (14) obey the relations : $|x(n)|=O(n)$ and $|x(n+1)-x(n)|=O(1)$, as $n \rightarrow \infty$.

\title{
REFERENCES
}

[1] B. Szmanda, Nonoscillation, oscillation and growth of solutions of nonlinear difference equations of second order, J. Math. Anal. Appl., 109 (1985), 22-30.

[2] E.H. YANG, Boundedness conditions for solutions of the differential equation $\left(a(t) x^{\prime}\right)^{\prime}+f(t, x)=0$, Nonlinear Anal., 8 (1984), 541-548.

[3] L. Hatvani, On the asymptotic behavior of $\left(p(t) x^{\prime}\right)^{\prime}+q(t) f(x)=0$, Publ. Math. Debrecen, 19 (1972), 225-237.

[4] V.B. Demidovic, A stability test for difference equations, Differentsial'nye Uravneniya, 5 (1969), 1247-1255 (in Russian).

[5] F. MENG. Boundedness of solutions of a class of higher order difference equations, Ann. Differential Equations, 4 (1988), 451-460.

\author{
Department of Mathematics \\ Linyi Teacher's College \\ LiNyi SHANDONG \\ P.R. CHINA \\ Department of Mathematics \\ QUFU NORMAL UNIVERSITY \\ Qufu, SHANDONG 273165 \\ P. R. CHINA \\ Department of Mathematics \\ QUFu NORMal UNIVERSITY \\ Qufu, Shandong 273165 \\ P.R. CHINA
}

